\title{
Reliability assessment for multi-state systems under uncertainties based on the
} Dempster-Shafer theory

\author{
Mohamed Sallak, Walter Schön, and Felipe Aguirre \\ Compiegne University of Technology, UMR CNRS 7253 \\ Heudiasyc BP 20529, 60205 Compiegne cedex, France \\ \{mohamed.sallak, walter.schon, felipe.aguirre-martinez\}@utc.fr
}

\begin{abstract}
This paper presents an original method for evaluating reliability indices for Multi-State Systems (MSSs) in the presence of aleatory and epistemic uncertainties. In many real world MSSs an insufficiency of data makes it difficult to estimate precise values for component state probabilities. The proposed approach applies the Transferable Belief Model (TBM) interpretation of the Dempster-Shafer theory to represent component state beliefs and to evaluate the MSS reliability indices. We use the example of an oil transmission system to demonstrate the proposed approach and we compare it with the Universal Generating Function method. The value of the Dempster-Shafer theory lies in its ability to use several combination rules in order to evaluate reliability indices for MSSs that depend on the reliability of the experts' opinions as well as their independence.
\end{abstract}

\section{KEY WORDS}

Multi-State System (MSS), reliability indices, Dempster-Shafer (D-S) theory, Transferable Belief Model (TBM), Experts' opinion.

\section{INTRODUCTION}

In traditional binary reliability both systems and components have only two possible states: perfect functioning and complete failure [9], [25]. However, a system and its components can have different states 
characterized by different levels of performance. Such systems are referred to as Multi-State Systems (MSSs). For example, a power station may have states $0,1,2,3$ and 4 that correspond to generating electricity at $0,25,50,75$ and 100 percent of its full capacity [61]. Therefore, reliability analysis of MSSs is much more complex than for binary state systems.

MSS theory has been a subject of investigation since 1978 in [8], [21]. A comprehensive presentation of MSS reliability theory and its applications can be found in the first book devoted to the reliability analysis of MSSs [35]. A recent review of the literature can be found in [39]. Practical methods of MSS reliability assessment are based on four different approaches [17], [43]: the structure function [44], [58], the Monte Carlo simulation technique [45], [69], the Markov approach [34], [62] and the Universal Generating Function (UGF) method [17], [31], [32]. The structure function approach based on the extension of Boolean models to multi-valued models was the first method to be developed for MSS reliability assessment. Monte Carlo simulation can represent any real world problem for the purposes of reliability assessment. In order to use the Markov method we need to generate all the possible states of a system. However, the number of states can be extremely large, even for a relatively small number of system elements. The UGF method was introduced in [57] and proved to be very effective in evaluating the reliability of different types of MSSs [35]. The UGF function extends the moment-generating function and allows the entire set of MSS performances to be obtained, based on the performance of its components for several system configurations. This can be done by introducing different composition operators over the UGF functions. [56] presents an interesting comparison of the four approaches.

Conventional MSS reliability theory makes two fundamental assumptions [17]: (i) every state probability of an MSS element can be fully characterized by probability measures; and (ii) the performance rate of any MSS element can be precisely determined. However, for some MSSs, there are different types of uncertainties about the state probabilities and performance rates of elements [12]. In recent years a general Fuzzy Multi-State Systems (FMSS) approach has been proposed to handle uncertainties in reliability assessments of MSSs [19]. This approach assumes that the state probabilities and/or the state performances of components can be represented by fuzzy values [18], [19], whereas the D-S approach developed in this paper assumes that the state beliefs of components are represented by belief functions and that the state performances of components are discrete values. The literature includes several attempts to extend belief functions to fuzzy events. The first extension of D-S theory to the general framework of 
fuzzy set theory was proposed by Zadeh in the context of information granularity and possibility theory [67]. A number of different generalizations were then proposed, according to how a measure of inclusion among fuzzy sets is used to define the belief functions [26], [36], [41], [52], [63], [65], [66]. In order to take fuzzy numbers into account, fuzzy numbers can be assigned to each focal element of the belief structure. This may be achieved by considering the upper and lower bounds of $\alpha$-cuts of fuzzy numbers. The structure obtained, introduced by Denœux [15], is called a Fuzzy-Valued Belief Structure (FVBS). This structure is defined as a fuzzy set of belief structures on the frame of discernment $\Omega$, whose belief masses are restricted by fuzzy numbers. Fuzzy credibility and plausibility can then be evaluated.

In this paper we are only interested in uncertainties about the state probabilities of elements. Uncertainties are classified into two categories: aleatory uncertainty and epistemic uncertainty. Aleatory uncertainty is the inherent variation associated with the physical system or the environment, such as the inherent variability of component failure. It is referred to as variability, random uncertainty, and stochastic uncertainty [40]. Knowledge provided by experts cannot be expected to reduce aleatory uncertainty. This type of uncertainty is thus also known as irreducible uncertainty. Epistemic uncertainty is an uncertainty that is due to a lack of knowledge of quantities or processes within the system or the environment. It is also referred to as reducible uncertainty or subjective uncertainty [27], [37]. Epistemic uncertainty can be eliminated by obtaining knowledge that was originally lacking, and expert opinion may be useful here. Over the last few years the risk assessment community has generally held that distinguishing between these types of uncertainty is useful and important when evaluating the reliability of systems [5]. In case of large amount of reliability data, the classical probabilistic approach was widely used to manage uncertainties in risk and reliability assessments [4]-[6]. This approach was based on the definition given by Laplace of the probability of an event as the ratio of the number of cases favorable to it, to the number of all possible cases when all cases are equally possible [30]. The frequentist probabilistic approach introduced by Venn [59] which defined the event probability as the limit of its relative frequency in a large number of trials was also widely used [4]-[6]. However, in the case of components that fail only rarely (nuclear systems, chemical processes, railway systems, etc.) or components that have not been operated long enough to generate a sufficient quantity of reliability data, expert judgment is required and both classical and frequentist probabilistic approaches become not suitable in these cases [4]-[6], [46]. For this reason, several methods were proposed to manage uncertainties such as Bayesian approach, interval approach, 
evidence theory, possibility theory, etc.

The Bayesian approach was based on the use of subjective probabilities to represent expert judgment [3]. The subjective probabilities of an event indicate the degree to which the expert believes it [23]. The probability distributions representing the aleatory uncertainties are proposed such as for example the representation of a lifetime component by an exponential distribution. The epistemic uncertainties about the parameter values of the distributions are then represented by prior subjective probability distributions. The equation of Bayes is used to compute the new epistemic uncertainties in terms of the posterior distributions in case of new reliability data. Finally, the predictive distributions of the quantities of interest such as the lifetime of new components are derived by using the total probability law [3], [22], [42], [68]. The predictive distributions are subjective but they also take into account the aleatory uncertainties represented by the prior probability models. However, There are some critics about the Bayesian approach exposed particularly by Walley [60] and Caselton and Luo [11]. In a situation of ignorance a Bayesian approach must equally allocate subjective probabilities over the frame of discernment. Thus there is no distinction between uncertainty and ignorance.

The D-S theory also known as the evidence theory or belief functions theory is a generalization of the Bayesian theory of subjective probability. Whereas the Bayesian theory requires probabilities for each question of interest, belief functions allow us to base degrees of belief for one question on probabilities for a related question [50]. To illustrate the idea of obtaining degrees of belief for one question from subjective probabilities for another, we propose an example in risk assessment inspired from the example of limb given by Shafer [50]. Suppose we have subjective probabilities for the reliability of a risk expert A. The probability that $\mathrm{A}$ is reliable is 0.75 , and the probability that $\mathrm{A}$ is not reliable is 0.25 . The risk expert A reports us that a component $\mathrm{i}$ is failed. This information which must be true if A is reliable, is not necessarily false if $\mathrm{A}$ is not reliable. The risk expert testimony justifies a 0.75 degree of belief that the component $\mathrm{i}$ is failed, but only a 0 degree of belief (not a 0.25 degree of belief) that the component $i$ is not failed. This value does not mean that we are sure that the component $\mathrm{i}$ is failed, as a zero probability would. It means that the risk expert's testimony gives us no reason to believe that the component $\mathrm{i}$ is failed. The 0.75 and the 0 constitute a belief function. Thus there is no requirement that belief not committed to a given proposition should be committed to its negation. The second important issue in D-S theory is that belief measures of uncertainty may be assigned to overlapping sets and subsets of 
hypotheses, events or propositions as well as to individual hypothesis. There are several interpretations of D-S theory (Dempster's model [14], the Theory of Hints [28], etc.). In this work the Transferable Belief Model (TBM) interpretation of the D-S theory is proposed.

The paper is organized as follows: Section 2 introduces basic concepts of D-S theory and TBM. Section 3 presents and discusses the proposed MSS model based on the TBM, the corresponding structure function and reliability indices. Section 4 presents an example comparing the UGF method and the proposed approach in the case of aleatory uncertainty. The same example with expert opinion is then studied using the TBM in presence of both aleatory and epistemic uncertainties. Finally section 5 presents some conclusions.

\section{BACKGROUND OF D-S THEORY AND TBM}

D-S is a theory for uncertain reasoning under both aleatory and epistemic uncertainties. It was first developed by Dempster [14] and extended by Shafer [50]. The first work using D-S theory in reliability assessment was presented by Dempster and Kong [13]. In recent years D-S theory has been used by numerous researchers to quantify uncertainty in the reliability analysis of binary state systems [1], [7], [33], [48], [49], [51], [55]. Several different interpretations of D-S theory have been put forward: Dempster's model [14], the Theory of Hints [28], the probability of modal propositions model [47] and the Transferable Belief Model (TBM) [54]. Each model corresponds to a different understanding of the concept of uncertainty. The interpretation of D-S theory that we have adopted in this paper is based on the TBM, which is a model developed outside the scope of probability theory and can thus avoid the accusation that D-S theory is understood as a special form of upper and lower probability theory [5].

\section{A. Basic Belief Assignment (BBA)}

The definition domain of the variable of interest $x$ is called the frame of discernment $\Omega$, where all of the possible events are mutually exclusive elementary propositions. As an example, let us consider a frame of discernment $\Omega=\left\{x_{1}, x_{2}\right\}$, meaning that $x_{1}$ and $x_{2}$ are elementary propositions and mutually exclusive of each other. The power set $2^{\Omega}$ is the set of all the subsets of $\Omega$ including itself, i.e.: $2^{\Omega}=$ $\left\{\{\emptyset\},\left\{x_{1}\right\},\left\{x_{2}\right\}, \Omega\right\}$. A Basic Belief Assignment (BBA) on $\Omega$ is a function $m^{\Omega}: 2^{\Omega} \rightarrow[0,1]$ which maps belief masses onto events or sets of events such that: 


$$
\sum_{A \in 2^{\Omega}} m^{\Omega}(A)=1
$$

An agent holding a piece of evidence allocates unitary amounts of belief to the different subsets of $\Omega$. The number $m^{\Omega}(A)$ represents the support to A given by the agent's belief [53]. There is a distinction between probabilities and BBAs: probability distribution functions are defined on $\Omega$, whereas BBAs are defined on the power set $2^{\Omega}$. This means that there are $2^{\operatorname{card}(\Omega)}$ possible hypotheses in D-S theory, while in probability theory there are only $\operatorname{card}(\Omega)$ possible hypotheses. Furthermore, the sub-additivity hypothesis is not required in D-S theory like it is in probability theory.

The subsets $A \subset \Omega$ such that $m^{\Omega}(A)>0$ are called focal sets of $m^{\Omega}$. Full knowledge is represented by a BBA having a singleton $\{x\}(x \in \Omega)$ as a unique focal set. A Bayesian BBA is a special case where all of the focal sets are singletons and is equivalent to probabilities. Complete ignorance is represented by a BBA having only one focal element equal to $\Omega$ and which is termed vacuous.

According to Klir and Folger [24], the BBAs have some important properties which distinguishes it from being a probability function:

- It is not required that $m(\Omega)=1$.

- It is not required that $m(A) \leq m(B)$ when $A \subseteq B$.

- No relationship between $m(A)$ and $m(\bar{A})$.

- Also $m(A)+m(\bar{A})$ does not always have to be 1 .

For simplicity, let us consider a component $i$ with two states. The frame of discernment $L_{i}$ of the component $i$ is then given by $L_{i}=\left\{0_{i}, 1_{i}\right\}$, where $0_{i}$ and $1_{i}$ denote respectively the failed and operational states of the component $i$. If an expert asserts a portion of belief $x_{i}$ that the component $i$ is working at time $t$ and a portion of belief $y_{i}$ that the component $i$ is not working at time $t$, this will be expressed as follows: $m_{\text {Expert }}^{L_{i}}\left(\left\{1_{i}\right\}\right)=x_{i}$ and $m_{\text {Expert }}^{L_{i}}\left(\left\{0_{i}\right\}\right)=y_{i}$. The epistemic uncertainty is represented by $m_{\text {Expert }}^{L_{i}}\left(\left\{0_{i}, 1_{i}\right\}\right)=1-x_{i}-y_{i}$. 


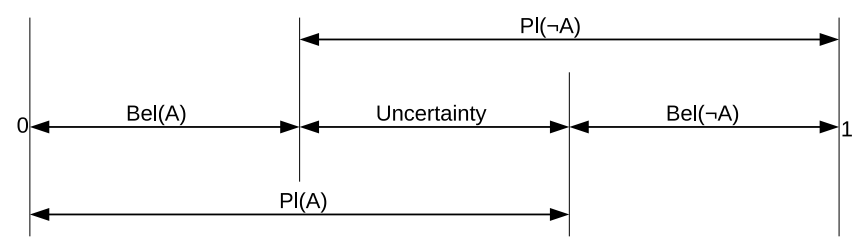

Fig. 1: The belief interval [29]

\section{B. Belief and plausibility functions}

The most important measures of uncertainty provided by D-S theory are known as belief and plausibility functions. The belief $\mathrm{Bel}$ and plausibility $\mathrm{Pl}$ functions for a subset $A$ are defined as follows:

$$
\begin{aligned}
& \operatorname{Bel}(A)=\sum_{B \subseteq A} m^{\Omega}(B) \quad \forall A \subseteq \Omega, \forall B \subseteq \Omega \\
& P l(A)=\sum_{B \cap A \neq \emptyset} m^{\Omega}(B) \quad \forall A \subseteq \Omega, \forall B \subseteq \Omega
\end{aligned}
$$

$\operatorname{Bel}(A)$ is obtained by adding the BBAs of propositions that totally agree with $A$, whereas $P l$ is obtained by adding the BBAs of propositions that agree with $A$ totally and partially (cf. Figure 1). $[\operatorname{Bel}(A), P l(A)]$ is the interval that describes the uncertainty of $A$. The functions $B e l$ and $P l$, although they are also functions mapping events $\mathrm{A}$ into $[0,1], \emptyset$ into 0 and $\Omega$ into 1 , do not fulfill in the general case the sub-additivity properties given for probability. They are related to each other by the following equation:

$$
\operatorname{Pl}(A)+\operatorname{Bel}(\bar{A})=1
$$

where $\bar{A}$ represents the negation of the event A.

Let us consider the same component $i$. The belief measure concerning the functioning of component $i$ at time $t$ is given by: $\operatorname{Bel}\left(\left\{1_{i}\right\}\right)=m_{\text {Expert }}^{L_{i}}\left(\left\{1_{i}\right\}\right)$ and the plausibility measure is given by: $\operatorname{Pl}\left(\left\{1_{i}\right\}\right)=$ $m_{\text {Expert }}^{L_{i}}\left(\left\{1_{i}\right\}\right)+m_{\text {Expert }}^{L_{i}}\left(\left\{0_{i}, 1_{i}\right\}\right)$. The availability of the component $i$ at time $t$ is then given by:

$$
A_{i}=\left[\underline{A_{i}}, \overline{A_{i}}\right]=\left[\operatorname{Bel}\left(\left\{1_{i}\right\}\right), \operatorname{Pl}\left(\left\{1_{i}\right\}\right)\right]
$$

The quantity $\underline{A_{i}}$ represents the total amount of justified support given to the proposition "the component 

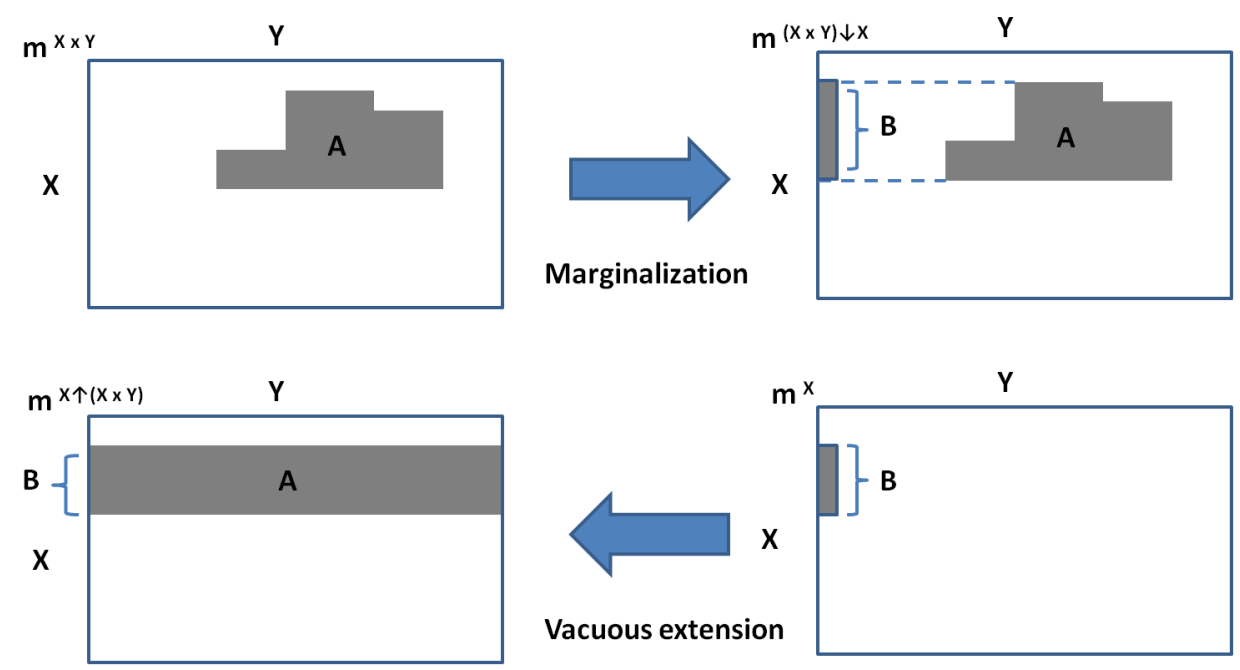

Fig. 2: Marginalization and vacuous extension operations

$i$ is available at time $\mathrm{t}$ ", while the quantity $\overline{A_{i}}$ represents the maximum amount of specific support that could be given to the proposition "the component $i$ is available at time t" if justified by additional information. Finally, the quantity $\overline{A_{i}}-\underline{A_{i}}$ represents the epistemic uncertainty.

\section{Marginalization and vacuous extension}

The first step in TBM is to define the frame of discernment. As noticed by Shafer [50], the degree of granularity of the frame is always to some extent a matter of convention, since any element representing a state of nature can always be split into several possibilities. Hence, it is fundamental to examine how a belief function defined on a frame may be expressed in a finer or, conversely, in a coarser frame.

Consider two finite sets $X$ and $Y$. A mapping $\rho: 2^{X} \rightarrow 2^{Y}$ is called a refining if it satisfies:

- $\rho(B)=\bigcup_{y \in B} \rho(\{y\}) \quad \forall B \subseteq Y$.

- The set $\{\rho(y), y \in Y\} \subseteq 2^{X}$ is a partition of $X$.

$Y$ is called a coarsening of $X$, and $X$ is called a refinement of $Y$. A BPA $m^{Y}$ on $Y$ can be transformed into a BBA $m^{X}$ on a refinement $X$ by transferring each mass $m^{Y}(B)$ for $B \subseteq Y$ to $m^{X}(A)$ for $A=\rho(B)$. This operation is called a vacuous extension of $m^{Y}$ to $X$ (cf. Figure 2). The inverse operation is the marginalization (cf. Figure 2). The formulas of vacuous extension and marginalization are given in the Appendix. 


\begin{tabular}{llll}
\hline & $\left\{0_{i}\right\}$ & $\left\{1_{i}\right\}$ & $\left\{0_{i}, 1_{i}\right\}$ \\
\hline Expert 1 & 0.1 & 0.3 & 0.6 \\
Expert 2 & 0 & 0.4 & 0.6 \\
Expert 1 $\oplus$ Expert 2 & 0.0625 & 0.5625 & 0.3750 \\
\hline
\end{tabular}

TABLE I: Dempster combination of BBAs

\section{Combination rules}

The D-S evidence theory can aggregate multiple sources of information through the combination rules. The two most familiar rules of combination are the Conjunctive and Disjunctive rules [50] (the formulas for the different rules can be found in the Appendix). Dempster's rule is a widely-used rule that consists in applying a conjunctive combination followed by a normalization of the conflict factor $k$ (the formula for Dempster's rule can be found in the Appendix). This conflict factor is equivalent to the BBA allocated to the empty set.

Let us suppose that Expert 1 asserts a 0.3 portion of belief that component $i$ is working and a 0.1 portion of belief that it is not working at time $t$. Expert 2 asserts a 0.4 portion of belief that component $i$ is working at time $t$ and no belief at all that it is not working. Dempster's rule of combination applied to the BBAs from experts 1 and 2 gives the new BBAs shown in Table I. Also, since BBAs have been combined the level of conflict $k$ can be gauged. In this case it is $k=0.04$, indicating a minor conflict in the evidence from the two experts.

Several other combination rules have been defined in D-S theory that depend on the reliability of experts and the conflict between them (Yager rule [64], Dubois and Prade rule [20], Cautious rule [16], etc.). For more details, see [14], [16], [50], [53].

\section{TBM OF MUlTi-STATE Systems}

\section{A. General model}

Let us consider a system $S$ with $n$ components. For $i=1, \ldots, n, X_{i}$ denotes the state of the $i$-th component. The set $L_{i}=\left\{0,1, \ldots, m_{i}\right\}$ representing all states of the $i$-th component is linearly ordered $\left(L_{i}, \leq\right)$, i.e. there exists a linear order (total, antisymmetric, transitive binary relation) over each set $L_{i}$. Complete failure (the worst state) corresponds to state 0 . Perfect functioning (the best state) corresponds to state $m_{i}$. A general model of MSS with a partial ordering over the set $L_{i}$ was proposed by Montero 
et al. [38]. In D-S theory, $L_{i}$ can be considered as the frame of discernment of the $i$-th component. The state vector of the MSS is $X=\left(X_{1}, X_{2}, \ldots, X_{n}\right)$.

A central problem in reliability theory is how to determine the relationship between the system states and the states of its components. This relationship can be described by a structure function defined by the mapping $\varphi: L_{1} \times L_{2} \times \ldots \times L_{n} \rightarrow L_{S}$. The value $\varphi\left(X_{1}, X_{2}, \ldots, X_{n}\right)$ is the system state when each component $i$ is at state $X_{i}$. The structure function $\varphi$ is assumed to be non-decreasing, i.e.:

$$
X \leq Y \Rightarrow \varphi(X) \leq \varphi(Y)
$$

This means that improving the state of one or more components cannot lead to a lower system performance. We also assume that

$$
\varphi(0,0, \ldots, 0)=0 \quad \text { and } \quad \varphi\left(m_{1}, m_{2}, \ldots, m_{n}\right)=m_{S}
$$

The BBAs of the $i$-th component states are defined by the mapping $m^{L_{i}}: 2^{L_{i}} \rightarrow[0,1]$ such that

$$
\sum_{A \in 2^{L_{i}}} m^{L_{i}}(A)=1
$$

Let $m_{\{j\}}^{L_{i}}=m^{L_{i}}\left(X_{i}=\{j\}\right)$ denote the BBA that the $i$-th component is in state $j$. The BBA $m_{\{j, k\}}^{L_{i}}=$ $m^{L_{i}}\left(X_{i}=\{j, k\}\right)$ represents the fact that the $i$-th component is in state $j$ or $k$.

For $j=0, \ldots, m_{i}$, the vacuous extension is used to extend the BBAs $m^{L_{i}}$ of each component $i$ to the product space $L_{1} \times L_{2} \ldots \times L_{n} \times L_{S}$. The resulting BBAs of all components are combined using Dempster's rule. The resulting BBAs are then combined with the BBA representing the system configuration. The BBA $m_{\text {Conf }}^{L_{1} \times L_{2} \ldots \times L_{n} \times L_{S}}$ represents the relation between the state of system and the state of its components. To obtain the BBAs of system states the final results are marginalized on $L_{S}$ :

$$
\begin{aligned}
m^{L_{S}}= & \left(m^{L_{1} \uparrow L_{1} \times L_{2} \ldots \times L_{n} \times L_{S}} \oplus m^{L_{2} \uparrow L_{1} \times L_{2} \ldots \times L_{n} \times L_{S}} \oplus \ldots\right. \\
& \left.\ldots \oplus m^{L_{n} \uparrow L_{1} \times L_{2} \ldots \times L_{n} \times L_{S}} \oplus m_{\text {Config }}^{L_{1} \times L_{2} \ldots \times L_{n} \times L_{S}}\right)^{\downarrow L_{S}}
\end{aligned}
$$

For example, let us consider a system $S$ with two components 1 and 2. For simplicity we consider that the system and its components have two states $0_{i}$ and $1_{i}$. We use the vacuous extension to extend $m^{L_{1}}$ and $m^{L_{2}}$ to the product space $L_{1} \times L_{2} \times L_{S}$ and we combine the obtained BBAs using the Dempster 
rule. The resulting BBAs are combined with $m_{\text {Config }}^{L_{1} \times L_{2} \times L_{S}}$.

For serial configuration (a failure of any component 1 or 2 results in failure of the entire system $S$ ) we have $m_{\text {Series }}^{L_{1} \times L_{2} \times L_{S}}\left(\left\{\left(1_{1}, 1_{2}, 1_{S}\right),\left(0_{1}, 0_{2}, 0_{S}\right),\left(0_{1}, 1_{2}, 0_{S}\right),\left(1_{1}, 0_{2}, 0_{S}\right)\right\}\right)=1$.

For parallel configuration (at least one of the two components 1 or 2 must succeed for the system $S$ to succeed) we have $m_{\text {Parallel }}^{L_{1} \times L_{2} \times L_{S}}\left(\left\{\left(1_{1}, 1_{2}, 1_{S}\right),\left(0_{1}, 0_{2}, 0_{S}\right),\left(0_{1}, 1_{2}, 1_{S}\right),\left(1_{1}, 0_{2}, 1_{S}\right)\right\}\right)=1$.

To obtain the BBAs of the system $S$ the final result is marginalized on $L_{S}$.

Belief and plausibility functions are then computed using Eq. 1 and Eq. 2. Finally, the lower and upper bounds (belief interval) for the system to be in state $j$ are given by:

$$
h_{j}=\left[\underline{h_{j}}, \overline{h_{j}}\right]=[\operatorname{Bel}(\varphi(X)=j), P l(\varphi(X)=j)] \quad j=0, \ldots, m_{S}
$$

System utility is an important performance measure introduced by Aven [2] for the study of multi-state systems. At each state $j$ an MSS yields a particular gain. This gain is termed the system utility at state $j$ and is denoted $u_{j}$. The overall system utility is the expected utility of the system, defined as follows:

$$
U=[\underline{U}, \bar{U}]=\left[\sum_{j=0}^{m_{S}} u_{j} \cdot \operatorname{Bel}(\varphi(X)=j), \sum_{j=0}^{m_{S}} u_{j} \cdot \operatorname{Pl}(\varphi(X)=j)\right] \quad j=0, \ldots, m_{S}
$$

The overall system utility of the system $S$ is defined as follows:

$$
U=\left[u_{0} \cdot \operatorname{Bel}(\varphi(X)=0)+u_{1} \cdot \operatorname{Bel}(\varphi(X)=1), u_{0} \cdot \operatorname{Pl}(\varphi(X)=0)+u_{1} \cdot \operatorname{Pl}(\varphi(X)=1)\right]
$$

where belief and plausibility functions are given by:

$$
\begin{gathered}
\operatorname{Bel}(\varphi(X)=0)=m^{L_{S}}\left(\left\{0_{S}\right\}\right) \\
\operatorname{Bel}(\varphi(X)=1)=m^{L_{S}}\left(\left\{1_{S}\right\}\right) \\
\operatorname{Pl}(\varphi(X)=0)=m^{L_{S}}\left(\left\{0_{S}\right\}\right)+m^{L_{S}}\left(\left\{0_{S}, 1_{S}\right\}\right) \\
\operatorname{Pl}(\varphi(X)=1)=m^{L_{S}}\left(\left\{1_{S}\right\}\right)+m^{L_{S}}\left(\left\{0_{S}, 1_{S}\right\}\right)
\end{gathered}
$$




\section{B. MSS reliability indices}

MSS reliability measures were first introduced by Aven [2] and subsequently by Brunelle and Kapur [10]. In this work we consider the three measures most commonly used by reliability engineers:

1) MSS availability $A$.

2) MSS expected performance $E_{d}$.

3) MSS unsupplied demand $E_{u}$.

The aim of this section is to define the above reliability measures in the TBM framework. The set $g_{i}=\left\{g_{i 0}, g_{i 1}, \ldots, g_{i m_{i}}\right\}$ represents the performance rates of component $i$ at states $j\left(j=\left\{0, \ldots, m_{i}\right\}\right)$. The set $G=\left\{G_{0}, G_{1}, \ldots, G_{m_{S}}\right\}$ represents the performance rates of the system. We define MSS availability $A$ as the belief that the MSS will be in a state with a performance level greater than or equal to the demand $w$. MSS availability is given by:

$$
A(w)=[\operatorname{Bel}(\varphi(X) \geq j), P l(\varphi(X) \geq j)]
$$

where $j$ corresponds to the first state which satisfies the demand $w$.

Another important measure of system performance is the MSS expected output performance $E_{d}$. This index determines the system's expected performance, and does not depend on the demand $w$. Therefore, $E_{d}$ defines the average capacity (productivity) of the system. It can be obtained as follows:

$$
E_{d}=\left[\sum_{j=0}^{m_{S}} \operatorname{Bel}(\varphi(X)=j) \cdot G_{j}, \sum_{j=0}^{m_{S}} \operatorname{Pl}(\varphi(X)=j) \cdot G_{j}\right]
$$

In some cases the expected unsupplied demand $E_{u}$ may be used as a measure of system output performance. This index can be formulated as:

$$
E_{u}(w)=\left[\sum_{j=0}^{m_{S}} \operatorname{Bel}(\varphi(X)=j) \cdot \max \left(w-G_{j}, 0\right), \sum_{j=0}^{m_{S}} \operatorname{Pl}(\varphi(X)=j) \cdot \max \left(w-G_{j}, 0\right)\right]
$$

It should be noted that the MSS expected output performance $E_{d}$ and MSS expected unsupplied performance $E_{u}$ are particular cases of the overall system utility defined in Eq.6 when the system utility functions $u_{j}$ at state $j$ are respectively equal to $G_{j}$ and $\max \left(w-G_{j}, 0\right)$. 


\section{Construction of BBAs}

1) From observations: Let us consider $n$ observations of events or sets of events in the frame of discernment $\Omega=\left\{A_{1}, \ldots, A_{n}\right\}$. Let $c_{i}$ denote the number of occurrences of an event $A_{i}$. The BBAs related to the occurrence of $A_{i}$ can then be obtained as follows:

$$
m^{\Omega}\left(\left\{A_{i}\right\}\right)=\frac{c_{i}}{n}
$$

If we lack any observations relating to the occurrence of the event $A_{i}$, then if $c_{i, j}$ denotes the number of occurrences of a set of events $\left\{A_{i}, A_{j}\right\}$, we obtain

$$
m^{\Omega}\left(\left\{A_{i}, A_{j}\right\}\right)=\frac{c_{i, j}}{n}
$$

The $B e l$ and $P l$ functions are then computed from BBAs using Eq. 1 and Eq. 2. For example, let us consider a system with two components 1 and 2. The two components can have three states, i.e. $L_{1}=\{0,1,2\}$ and $L_{2}=\{0,1,2\}$. Out of 50 observations we have 30 observations indicating that component 1 is in state 0 or 1,10 observations indicating that it is in state 2 and a further 10 indicating that it is in state 0,1 , or 2 . At the same time 32 observations indicate that component 2 is in state 0,8 observations indicate that it is in state 1 and 10 observations indicate that this component 2 is in states 0,1 or 2 . This is written as:

$$
\begin{gathered}
m_{\{0,1\}}^{L_{1}}=30 / 50=0.6 \\
m_{\{2\}}^{L_{1}}=10 / 50=0.2 \\
m_{\{0,1,2\}}^{L_{1}}=10 / 50=0.2 \\
m_{\{0\}}^{L_{2}}=32 / 50=0.64 \\
m_{\{1\}}^{L_{2}}=8 / 50=0.16 \\
m_{\{0,1,2\}}^{L_{2}}=10 / 50=0.2
\end{gathered}
$$

Using Eq.1 and Eq.2 the components' Bel and Pl functions (belief intervals) for being in each of these different states are computed (cf. Table II). 


\begin{tabular}{lll}
\hline States & $\begin{array}{l}\text { Component 1 } \\
\text { [Bel, Pl] }\end{array}$ & $\begin{array}{l}\text { Component 2 } \\
\text { [Bel, Pl] }\end{array}$ \\
\hline$\{0\}$ & {$[0,0.8]$} & {$[0.64,0.84]$} \\
$\{1\}$ & {$[0,0.8]$} & {$[0.16,0.36]$} \\
$\{2\}$ & {$[0.2,0.4]$} & {$[0,0.2]$} \\
$\{0,1\}$ & {$[0.6,0.8]$} & {$[0.8,1]$} \\
$\{0,2\}$ & {$[0.2,1]$} & {$[0.64,0.84]$} \\
$\{1,2\}$ & {$[0.2,1]$} & {$[0.16,0.36]$} \\
$\{0,1,2\}$ & 1 & 1 \\
\hline
\end{tabular}

TABLE II: Belief intervals of components 1 and 2

\begin{tabular}{llll}
\hline & & \multicolumn{2}{c}{ Component 3 } \\
States & Expert 1 & Expert 2 & Expert 3 \\
& & & \\
\hline 0$\}$ & 0.3 & 0.4 & 0.45 \\
$\{1\}$ & 0.2 & 0.2 & 0.15 \\
$\{2\}$ & 0.1 & 0.2 & 0.25 \\
$\{0,1\}$ & 0 & 0 & 0 \\
$\{0,2\}$ & 0 & 0 & 0 \\
$\{1,2\}$ & 0 & 0 & 0 \\
$\{0,1,2\}$ & 0.4 & 0.2 & 0.15 \\
& & & \\
\hline
\end{tabular}

TABLE III: BBAs for component 3 according to experts' opinions

2) From expert opinion: Suppose that Expert 1 asserts a 0.3 portion of belief that component 3 is in state 0 , a 0.2 portion of belief that it is in state 1 and a 0.1 portion of belief that it is in state 2 (the remaining 0.4 represents ignorance and will be allocated to the frame of discernment). The corresponding values asserted by Expert 2 are 0.4, 0.2 and 0.2, while for Expert 3 they are 0.45, 0.15 and 0.25. The BBAs provided by the experts are shown in Table III, and the BBAs obtained from the combination rules in Table IV. Finally, the components' Bel and Pl functions for each state are given in Table V.

The conflict factor $k$ between BBAs is defined as follows:

$$
k=\sum_{A \cap B=\emptyset, \forall A \subseteq L_{i}, B \subseteq L_{j}} m^{L_{i}}(A) m^{L_{j}}(B)
$$

The factor $k$ is equal to 0.3, 0.4 and 0.32 respectively between Expert 1 and Expert 2, Expert 2 and Expert 3, and Expert 1 and Expert 3. Dempster's rule therefore does not lead to obvious contradictions, because the factor $k$ is not high. Dempster's rule assumes the three experts to be equally reliable and independent. The Disjunctive rule considers only one of the experts to be reliable, but we do not know 


\begin{tabular}{lll} 
States & Dempster's rule & Disjunctive rule \\
& & \\
$\{0\}$ & 0.6316 & 0.054 \\
$\{1\}$ & 0.1579 & 0.006 \\
$\{2\}$ & 0.1789 & 0.005 \\
$\{0,1\}$ & 0 & 0.12 \\
$\{0,2\}$ & 0 & 0.109 \\
$\{1,2\}$ & 0 & 0.037 \\
$\{0,1,2\}$ & 0.0316 & 0.669 \\
& & \\
\hline
\end{tabular}

TABLE IV: The obtained BBAs for component 3

\begin{tabular}{lll}
\hline & $\begin{array}{l}\text { Dempster's rule } \\
{[\mathrm{Bel}, \mathrm{Pl}]}\end{array}$ & $\begin{array}{l}\text { Disjunctive rule } \\
{[\mathrm{Bel}, \mathrm{Pl}]}\end{array}$ \\
\hline & & \\
$\{0\}$ & {$[0.6316,0.6632]$} & {$[0.0540,0.9520]$} \\
$\{1\}$ & {$[0.1579,0.1895]$} & {$[0.0060,0.8320]$} \\
$\{2\}$ & {$[0.1789,0.2105]$} & {$[0.0050,0.8200]$} \\
$\{0,1\}$ & {$[0.7895,0.8211]$} & {$[0.1800,0.9580]$} \\
$\{0,2\}$ & {$[0.8105,0.8421]$} & {$[0.1680,0.9570]$} \\
$\{1,2\}$ & {$[0.3368,0.3684]$} & {$[0.0480,0.9460]$} \\
$\{0,1,2\}$ & 1 & 1
\end{tabular}

TABLE V: Interval beliefs for component 3

which one. It is very important to choose a combination rule in accordance with the hypothesis under consideration (independence, reliability, conflict, etc.).

\section{NUMERICAL EXAMPLES}

\section{A. Example 1}

The TBM method proposed here has not been used before in MSS reliability assessment, and so we have chosen to compare it with the UGF method used by Ding and Lisianski for evaluating reliability indices in an oil transmission system [17]. In this example only aleatory uncertainty is considered (the components' state probabilities are precise values) [17].

The oil transmission system (cf. Figure 3) consists of three pipes. The oil flow is transmitted from point C to point E. The pipes' performance is measured by their transmission capacity (tons per minute). Elements 1 and 2 have three states. A state of total failure for both elements corresponds to a transmission capacity of zero, a state of partial failure corresponding to a capacity of 1 ton/min for element 1 and 1.5 tons $/ \mathrm{min}$ for element 2, and the operational state corresponds to capacities of 1.5 tons/min for element 1 and 2 


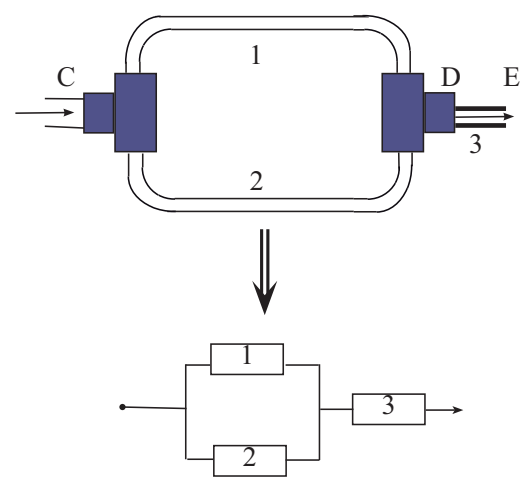

Fig. 3: The oil transmission system and its Reliability Block Diagram

tons/min for element 2, so that $g_{1}=\{0,1,1.5\}$ and $g_{2}=\{0,1.5,2\}$. The corresponding probabilities for element 1 are $p_{10}=0.1, p_{11}=0.1$, and $p_{12}=0.8$, and for element 2 are $p_{20}=0.1, p_{21}=0.2$, and $p_{22}=0.7$. Element 3 is binary. It has a state of total failure corresponding to a capacity of zero and a fully operational state with a capacity of 4 tons/min so that $g_{3}=\{0,4\}$. The corresponding probabilities are $p_{30}=0.04$ and $p_{31}=0.96$. The system output performance rate is defined as the maximum flow that can be transmitted from $\mathrm{C}$ to $\mathrm{E}$.

The total flow between points $\mathrm{C}$ and $\mathrm{D}$ through the parallel pipes 1 and 2 is equal to the sum of the flows in the two pipes. The flow from point $\mathrm{D}$ to point $\mathrm{E}$ is limited by the transmission capacity of element 3. This flow cannot however be greater than the flow between points $\mathrm{C}$ and $\mathrm{D}$. Therefore, the flow between points $\mathrm{C}$ and $\mathrm{E}$ (the system performance) is:

$$
G=\Phi\left(g_{1}(t), g_{2}(t), g_{3}(t)\right)
$$

where $\Phi$ is the function which maps component performance rates into system performance rates, as shown in Table VI. The set $G=\{0,1,1.5,2,2.5,3,3.5\}$ represents the system performance rates (the oil transmission system has 7 states). We shall now evaluate the reliability indices of the oil transmission system using both the UGF and the TBM methods.

1) UGF Method: The UGF Method (u-functions) was introduced by Ushakov in the mid-1980s [57], and it has proved to be very effective in evaluating the reliability of different types of MSSs [31], [34], [35]. It involves intuitively simple recursive procedures combined with simplification techniques. The UGF method includes the following steps: 


\begin{tabular}{ccccccccccccccccccc}
\hline$g_{1}$ & 0 & 0 & 0 & 0 & 0 & 0 & 1 & 1 & 1 & 1 & 1 & 1 & 1.5 & 1.5 & 1.5 & 1.5 & 1.5 & 1.5 \\
$g_{2}$ & 0 & 0 & 1.5 & 1.5 & 2 & 2 & 0 & 0 & 1.5 & 1.5 & 2 & 2 & 0 & 0 & 1.5 & 1.5 & 2 & 2 \\
$g_{3}$ & 0 & 4 & 0 & 4 & 0 & 4 & 0 & 4 & 0 & 4 & 0 & 4 & 0 & 4 & 0 & 4 & 0 & 4 \\
$G=\Phi\left(g_{1}, g_{2}, g_{3}\right)$ & 0 & 0 & 0 & 1.5 & 0 & 2 & 0 & 1 & 0 & 2.5 & 0 & 3 & 0 & 1.5 & 0 & 3 & 0 & 3.5
\end{tabular}

TABLE VI: Performance rates of the oil transmission system

- Evaluation of individual u-functions for each element.

- Evaluation of the resulting u-function for the whole MSS using composition operators.

- Evaluation of MSS reliability measures.

The u-function representing the probability mass function (pmf) of a random discrete variable $X_{i}$ is given by

$$
u_{i}(z)=\sum_{j=0}^{m_{i}} a_{i j} z^{x_{i j}}
$$

where $x_{i j}$ are the $m_{i}+1$ possible values of $X_{i}$ and $a_{i j}$ is the probability that $X_{i}$ is equal to $x_{i j}$. The $\mathrm{u}$-function representing the pmf of a function $\Phi$ of $n$ independent random variables $X_{1}, X_{2}, \ldots, X_{n}$ is defined using a composition operator $\Omega_{\Phi}$ as follows:

$$
\begin{aligned}
U(z) & =\Omega_{\Phi}\left(u_{1}(z), u_{2}(z), \ldots, u_{n}(z)\right) \\
& =\Omega_{\Phi}\left(\sum_{j_{1}=0}^{m_{1}} a_{1 j_{1}} z^{x_{1 j_{1}}}, \sum_{j_{2}=0}^{m_{2}} a_{2 j_{2}} z^{x_{2 j_{2}}}, \ldots, \sum_{j_{n}=0}^{m_{n}} a_{n j_{n}} z^{x_{n j_{n}}}\right) \\
& =\sum_{j_{1}=0}^{m_{1}} \sum_{j_{2}=0}^{m_{2}} \ldots \sum_{j_{n}=0}^{m_{n}}\left(\prod_{i=1}^{n} a_{i j_{i}} z^{\Phi\left(x_{1 j_{1}}, x_{2 j_{2}}, \ldots, x_{n j_{n}}\right)}\right)
\end{aligned}
$$

Using performance rates $g_{i j}$ and the corresponding probabilities $p_{i j}(t)$ that component $i(i \in\{1, \ldots, n\})$ is in state $j\left(j \in\left\{0, \ldots, m_{i}\right\}\right)$, the u-function for component $i$ is defined as follows:

$$
u_{i}(z)=\sum_{j=0}^{m_{i}} p_{i j} z^{g_{i j}}
$$

The u-functions of the three elements of the oil transmission system are then given by:

$$
\begin{aligned}
u_{1}(z) & =p_{10} z^{g_{10}}+p_{11} z^{g_{11}}+p_{12} z^{g_{12}} \\
& =0.1 z^{0}+0.1 z^{1}+0.8 z^{1.5}
\end{aligned}
$$




$$
\begin{gathered}
u_{2}(z)=p_{20} z^{g_{20}}+p_{21} z^{g_{21}}+p_{22} z^{g_{22}} \\
=0.1 z^{0}+0.2 z^{1.5}+0.7 z^{2} \\
u_{3}(z)=p_{30} z^{g_{30}}+p_{31} z^{g_{31}} \\
=0.04 z^{0}+0.96 z^{4}
\end{gathered}
$$

The composition operators $\Omega_{\Phi_{S}}$ for serial elements, $\Omega_{\Phi_{P}}$ for parallel elements and $\Omega_{\Phi_{B}}$ for elements connected in a bridge structure are defined in [35], where corresponding recursive procedures for their computation were introduced for different types of systems.

Applying the operator $\Omega_{\Phi}$ with $\Phi\left(g_{1}(t), g_{2}(t), g_{3}(t)\right)$ over the u-functions of the different elements comprising the oil transmission system, we obtain:

$$
\begin{aligned}
U(z) & =\Omega_{\Phi}\left(u_{1}(z), u_{2}(z), u_{3}(z)\right) \\
& =\Omega_{\Phi_{S}}\left(\Omega_{\Phi_{P}}\left(u_{1}(z), u_{2}(z)\right), u_{3}(z)\right)
\end{aligned}
$$

The function $\Phi$ is defined by the type of connection between the elements. Here the function $\Phi_{S}$ is defined as the min function, and the function $\Phi_{P}$ is defined as the sum function:

$$
\begin{aligned}
\Phi\left(g_{1}, g_{2}, g_{3}\right) & =\Phi_{S}\left(\Phi_{P}\left(g_{1}, g_{2}\right), g_{3}\right) \\
& \left.=\min \left(\left(g_{1}+g_{2}\right), g_{3}\right)\right)
\end{aligned}
$$

The u-function $u_{4}(z)$ for elements 1 and 2 in parallel is determined as follows:

$$
\begin{aligned}
u_{4}(z) & =\Omega_{\Phi_{P}}\left(u_{1}(z), u_{2}(z)\right) \\
& =\sum_{j_{1}=0}^{2} \sum_{j_{2}=0}^{2} p_{1 j_{1}} p_{2 j_{2}} . z^{g_{1 j_{1}}+g_{2 j_{2}}} \\
& =0.01 z^{0}+0.01 z^{1}+0.1 z^{1.5}+0.07 z^{2}+0.02 z^{2.5}+0.23 z^{3}+0.56 z^{3.5}
\end{aligned}
$$


Based on these procedures, the resulting UGF for the entire MSS can be obtained as follows:

$$
\begin{aligned}
U(z) & =\Omega_{\Phi_{S}}\left(u_{4}(z), u_{3}(z)\right) \\
& =\sum_{j_{3}=0}^{1} \sum_{j_{4}=0}^{6} p_{4 j_{4}} p_{3 j_{3}} . z^{\min \left\{g_{4_{4}}, g_{3_{3}}\right\}} \\
& =0.0496 z^{0}+0.0096 z^{1}+0.096 z^{1.5}+0.0672 z^{2}+0.0192 z^{2.5}+0.2208 z^{3}+0.5376 z^{3.5}
\end{aligned}
$$

Applying the operators $\delta_{A}, \delta_{E}$ and $\delta_{C}$ (introduced in [35]) over the resulting u-function of the whole MSS, the following MSS reliability indices are obtained:

- The MSS availability $A(w)$ for the arbitrary demand constant $w$ can be obtained by:

$$
A(w)=\delta_{A}(U(z), w)=\delta_{A}\left(\sum_{j=0}^{m_{S}} p_{j} z^{G_{j}}, w\right)=\sum_{j=0}^{m_{S}} p_{j} 1\left(G_{j}-w \geq 0\right)
$$

where $G_{j}$ is the performance rate of system at state $j$ and $p_{j}$ is the corresponding probability of the system being in state $j$.

- MSS Expected output performance $E_{d}$ for the given $U(z)$ using the following $\delta_{E}$ :

$$
E_{d}=\delta_{E}(U(z))=\delta_{E}\left(\sum_{j=0}^{m_{S}} p_{j} z^{G_{j}}\right)=\sum_{j=0}^{m_{S}} p_{j} G_{j}
$$

- MSS performance deficiency $E_{u}(w)$ for the given $U(z)$ and constant demand $w$ using the following $\delta_{D}$ :

$$
E_{u}(w)=\delta_{D}(U(z), w)=\delta_{D}\left(\sum_{j=0}^{m_{S}} p_{j} z^{G_{j}}, w\right)=\sum_{j=0}^{m_{S}} p_{j} \max \left(w-G_{j}, 0\right)
$$

Using Eq. 11, Eq. 12 and Eq. 13, the availability, expected output performance and performance deficiency of the oil transmission system obtained for each demand $w$ are computed (cf. Table VII).

2) TBM method: The system consists of 3 components. Components 1 and 2 have 3 states $\{0,1,2\}$. Component 3 has 2 states $\{0,1\}$. The state vector of the system is $X=\left(X_{1}, X_{2}, X_{3}\right)$. The frames of discernment of the components and of the system are:

$$
\begin{aligned}
& L_{1}=\{0,1,2\} \\
& L_{2}=\{0,1,2\}
\end{aligned}
$$




\begin{tabular}{cccc}
\hline Demand $w$ & \multicolumn{3}{c}{ UGF } \\
& $A$ & $E_{d}$ & $E_{u}$ \\
& & & \\
1 & 0.9504 & 2.88 & 0.0496 \\
1.5 & 0.9408 & 2.88 & 0.0792 \\
2 & 0.8448 & 2.88 & 0.1568 \\
2.5 & 0.7776 & 2.88 & 0.268 \\
3 & 0.7584 & 2.88 & 0.3888 \\
3.5 & 0.5376 & 2.88 & 0.6200 \\
& & &
\end{tabular}

TABLE VII: Example 1: MSS reliability indices based on UGF method

\begin{tabular}{cccccccccccccccccccc}
\hline$X_{1}$ & 0 & 0 & 0 & 0 & 0 & 0 & 1 & 1 & 1 & 1 & 1 & 1 & 2 & 2 & 2 & 2 & 2 & 2 \\
$X_{2}$ & 0 & 0 & 1 & 1 & 2 & 2 & 0 & 0 & 1 & 1 & 2 & 2 & 0 & 0 & 1 & 1 & 2 & 2 \\
$X_{3}$ & 0 & 1 & 0 & 1 & 0 & 1 & 0 & 1 & 0 & 1 & 0 & 1 & 0 & 1 & 0 & 1 & 0 & 1 \\
$X_{S}=\varphi\left(X_{1}, X_{2}, X_{3}\right)$ & 0 & 0 & 0 & 2 & 0 & 3 & 0 & 1 & 0 & 4 & 0 & 5 & 0 & 2 & 0 & 5 & 0 & 6 \\
\hline
\end{tabular}

TABLE VIII: Structure function of the oil transmission system

$$
\begin{gathered}
L_{3}=\{0,1\} \\
L_{S}=\{0,1,2,3,4,5,6\}
\end{gathered}
$$

The corresponding BBAs for element 1,2 and 3 are $m_{\{0\}}^{L_{1}}=0.1, m_{\{1\}}^{L_{1}}=0.1, m_{\{2\}}^{L_{1}}=0.8, m_{\{0\}}^{L_{2}}=0.1$, $m_{\{1\}}^{L_{2}}=0.2, m_{\{2\}}^{L_{2}}=0.7, m_{\{0\}}^{L_{3}}=0.04$, and $m_{\{1\}}^{L_{3}}=0.96$.

The vacuous extension defined in Eq.15 is used to extend the BBAs for the separate components to the product space $L_{1} \times L_{2} \times L_{3} \times L_{S}$. The resulting BBAs are combined using Dempster's rule, and the new BBAs are then combined with the BBA $m_{\text {config }}$, which represents the structure function $\varphi$ of the oil transmission system (cf. Table VIII). This BBA is given by:

$$
\begin{aligned}
& m_{\text {Config }}\left(\left\{X_{1}=0, X_{2}=0, X_{3}=0, X_{S}=0\right\},\left\{X_{1}=0, X_{2}=0, X_{3}=1, X_{S}=0\right\}, \ldots\right. \\
& \left.\ldots,\left\{X_{1}=2, X_{2}=2, X_{3}=0, X_{S}=0\right\},\left\{X_{1}=2, X_{2}=2, X_{3}=1, X_{S}=6\right\}\right)=1
\end{aligned}
$$

The resulting BBAs are then marginalized to the frame of discernment $L_{S}$ using Eq.14. Belief and plausibility functions are computed using Eq.1 and Eq.2, and the belief intervals of system states using Eq.4 and Eq.5 (cf. Table IX). Finally, using Eq.7, Eq.8 and Eq.9, we obtain the reliability indices summarized in Table X. In the absence of epistemic uncertainty we have $\operatorname{Bel}(\varphi(X)=j)=\operatorname{Pl}(\varphi(X)=j)$ 


\begin{tabular}{ccc}
\hline $\begin{array}{c}\text { System } \\
\text { states } j\end{array}$ & $\begin{array}{c}\text { Performance } \\
\text { rates (ton/min) }\end{array}$ & $h_{j}=\left[\underline{h_{j}, \overline{h_{j}}}\right]$ \\
\hline & & \\
0 & 0 & 0.0496 \\
1 & 1 & 0.0096 \\
2 & 1.5 & 0.096 \\
3 & 2 & 0.0672 \\
4 & 2.5 & 0.0192 \\
5 & 3 & 0.1536 \\
6 & 3.5 & 0.6048 \\
& & \\
\hline
\end{tabular}

TABLE IX: Example 1: Belief intervals of system states

\begin{tabular}{cccc}
\hline Demand $w$ & \multicolumn{3}{c}{ TBM } \\
& $A$ & $E_{d}$ & $E_{u}$ \\
\hline & & & \\
1 & 0.9504 & 2.88 & 0.0496 \\
1.5 & 0.9408 & 2.88 & 0.0792 \\
2 & 0.8448 & 2.88 & 0.1568 \\
2.5 & 0.7776 & 2.88 & 0.268 \\
3 & 0.7584 & 2.88 & 0.3888 \\
3.5 & 0.5376 & 2.88 & 0.6200
\end{tabular}

TABLE X: Example 1: MSS reliability indices based on the TBM method

for each state $\left.j \underline{\left(h_{j}\right.}=\overline{h_{j}}\right)$. Thus we obtain precise values instead of interval beliefs. As expected, the same reliability indices are obtained for the UGF and TBM methods (cf. Table VII and Table X).

\section{B. Example 2}

This example features the same oil transmission system used in Example 1, but epistemic uncertainty is also taken into account. The corresponding BBAs of component states are given by three experts (Expert 1, Expert 2 and Expert 3), as shown in Table XI. Three combination rules are used to aggregate the experts' opinions: Dempster's rule, the Disjunctive rule and the Cautious rule [16]. Table XII shows the BBAs of component states obtained using each of these combination rules.

Belief and plausibility functions are computed using Eq.1 and Eq.2. The belief intervals of system states for each rule are then computed using Eq.4 and Eq.5 (cf. Table XIII). As we can see, the Disjunctive rule gives a less precise belief interval than Dempster's rule and the Cautious rule.

The reliability indices of the system (cf. Table XIV) obtained for each demand $w$ using the three combination rules are computed using Eq.7, Eq.8 and Eq.9. 


\begin{tabular}{|c|c|c|c|c|c|c|c|c|c|}
\hline \multirow[t]{2}{*}{ Component $i$} & \multicolumn{3}{|c|}{ Expert 1} & \multicolumn{3}{|c|}{ Expert 2} & \multicolumn{3}{|c|}{ Expert 3} \\
\hline & $m_{\{0\}}$ & $m_{\{1\}}$ & $m_{\{2\}}$ & $m_{\{0\}}$ & $m_{\{1\}}$ & $m_{\{2\}}$ & $m_{\{0\}}$ & $m_{\{1\}}$ & $m_{\{2\}}$ \\
\hline 1 & 0.19 & 0.2 & 0.58 & 0.16 & 0.3 & 0.52 & 0.14 & 0.32 & 0.52 \\
\hline 2 & 0.15 & 0.24 & 0.56 & 0.18 & 0.29 & 0.53 & 0.10 & 0.26 & 0.6 \\
\hline 3 & 0.29 & 0.58 & 0 & 0.26 & 0.52 & 0 & 0.34 & 0.52 & 0 \\
\hline
\end{tabular}

TABLE XI: Example 2: BBAs of component states given by experts

\begin{tabular}{cccccccccc}
\hline \multirow{2}{*}{ Component $i$} & \multicolumn{4}{c}{ Dempster's rule } & \multicolumn{3}{c}{ Disjunctive rule } & \multicolumn{3}{c}{ Cautious rule } \\
& $m_{\{0\}}$ & $m_{\{1\}}$ & $m_{\{2\}}$ & $m_{\{0\}}$ & $m_{\{1\}}$ & $m_{\{2\}}$ & $m_{\{0\}}$ & $m_{\{1\}}$ & $m_{\{2\}}$ \\
& & & & & & & & & \\
1 & 0.0302 & 0.1196 & 0.8502 & 0.0043 & 0.0027 & 0.0256 & 0.1560 & 0.3144 & 0.5106 \\
2 & 0.0212 & 0.1064 & 0.8724 & 0.0192 & 0.0181 & 0.1568 & 0.1802 & 0.2907 & 0.5116 \\
3 & 0.2111 & 0.7798 & 0 & 0.1568 & 0.1781 & 0 & 0.3074 & 0.5662 & 0 \\
\hline
\end{tabular}

TABLE XII: Example 2: The obtained BBAs after combination

Dempster's rule is based on the assumption that the BBAs to be combined come from reliable experts. The expected output performance $E d=[2.572681,2.603106]$ never exceeds 3 tons/min and the system unsupplied demand ever exceeds $1 \mathrm{ton} / \mathrm{min}$. Suppose that the system safety standard requires that the system operation satisfies a required level of system availability greater than or equal to 0.6. This would imply that the oil transmission system cannot meet the system availability requirement if the demand $w$ is greater than $3(A=[0.578374,0.585170]$ for $w>3)$. The system unsupplied demand $E_{u}$ is always lower than 1 ton/min.

The Disjunctive rule merely assumes that at least one source of information is reliable, and we do not know which one. In the case where a system availability of $A>0.6$ is required we cannot reach a

\begin{tabular}{ccccc}
\hline $\begin{array}{c}\text { System } \\
\text { states } j\end{array}$ & $\begin{array}{c}\text { Performance } \\
\text { rates (ton/min) }\end{array}$ & $\begin{array}{c}\text { Dempster's rule } \\
{\left[\underline{h_{j}}, \overline{h_{j}}\right]}\end{array}$ & $\begin{array}{c}\text { Disjunctive rule } \\
{\left[\underline{h_{j}}, \overline{h_{j}}\right]}\end{array}$ & $\begin{array}{c}\text { Cautious rule } \\
{\left[\underline{h_{j}}, \overline{h_{j}}\right]}\end{array}$ \\
\hline & 0 & {$[0.211560,0.220665]$} & {$[0.025647,0.994379]$} & {$[0.325023,0.467572]$} \\
0 & 1 & {$[0.001981,0.002005]$} & {$[0.000008,0.968740]$} & {$[0.032853,0.175403]$} \\
1 & 1.5 & {$[0.016596,0.016795]$} & {$[0.000078,0.968810]$} & {$[0.076276,0.218825]$} \\
2 & 2 & {$[0.020564,0.029713]$} & {$[0.000119,0.968850]$} & {$[0.040901,0.183450]$} \\
3 & 2.5 & {$[0.009917,0.010038]$} & {$[0.000054,0.968786]$} & {$[0.052999,0.195549]$} \\
4 & 3 & {$[0.151858,0.153676]$} & {$[0.000981,0.969713]$} & {$[0.178829,0.321379]$} \\
5 & 3.5 & {$[0.578374,0.585170]$} & {$[0.004380,0.973112]$} & {$[0.150569,0.293119]$} \\
6 & & & & \\
\hline
\end{tabular}

TABLE XIII: Example 2: Belief intervals of system states 


\begin{tabular}{|c|c|c|c|}
\hline Demand & $A$ & $\begin{array}{c}\text { Dempster's rule } \\
E_{d}\end{array}$ & $E_{u}$ \\
\hline 1 & {$[0.779335,0.788440]$} & {$[2.572681,2.603106]$} & {$[0.211560,0.220665]$} \\
\hline 1.5 & {$[0.777354,0.786435]$} & {$[2.572681,2.603106]$} & {$[0.318331,0.332001]$} \\
\hline 2 & {$[0.760753,0.769645]$} & {$[2.572681,2.603106]$} & {$[0.433400,0.451734]$} \\
\hline 2.5 & {$[0.740150,0.748840]$} & {$[2.572681,2.603106]$} & {$[0.558751,0.581888]$} \\
\hline 3 & {$[0.730232,0.738807]$} & {$[2.572681,2.603106]$} & {$[0.689060,0.717062]$} \\
\hline 3.5 & {$[0.578374,0.585170]$} & {$[2.572681,2.603106]$} & {$[0.895299,0.929075]$} \\
\hline Demand & $A$ & $\begin{array}{l}\text { Disjunctive Rule } \\
E_{d}\end{array}$ & $E_{u}$ \\
\hline 1 & {$[0.052958,0.974353]$} & {$[0.018774,11.158645]$} & {$[0.025647,0.947042]$} \\
\hline 1.5 & {$[0.050538,0.974302]$} & {$[0.018774,11.158645]$} & {$[0.038475,1.749088]$} \\
\hline 2 & {$[0.028428,0.971658]$} & {$[0.018774,11.158645]$} & {$[0.051342,2.942482]$} \\
\hline 2.5 & {$[0.005416,0.967598]$} & {$[0.018774,11.158645]$} & {$[0.064268,4.528951]$} \\
\hline 3 & {$[0.005361,0.967259]$} & {$[0.018774,11.158645]$} & {$[0.077222,6.450237]$} \\
\hline 3.5 & {$[0.004380,0.931722]$} & {$[0.018774,11.158645]$} & {$[0.090666,8.841546]$} \\
\hline Demand & $A$ & $\begin{array}{c}\text { Cautious Rule } \\
E_{d}\end{array}$ & $E_{u}$ \\
\hline 1 & {$[0.532428,0.674977]$} & {$[1.425048,1.923970]$} & {$[0.325023,0.467572]$} \\
\hline 1.5 & {$[0.499575,0.642124]$} & {$[1.425048,1.923970]$} & {$[0.503961,0.789059]$} \\
\hline 2 & {$[0.423299,0.565848]$} & {$[1.425048,1.923970]$} & {$[0.721037,1.219959]$} \\
\hline 2.5 & {$[0.382398,0.524947]$} & {$[1.425048,1.923970]$} & {$[0.958563,1.742584]$} \\
\hline 3 & {$[0.329399,0.471948]$} & {$[1.425048,1.923970]$} & {$[1.222589,2.362983]$} \\
\hline 3.5 & {$[0.150569,0.293119]$} & {$[1.425048,1.923970]$} & {$[1.576030,3.144072]$} \\
\hline
\end{tabular}

TABLE XIV: Example 2: MSS reliability indices

decision, because for each demand $w$ the support of $A$ is very large. Moreover, the supports of $E_{d}$ and $E_{u}$ are also very large and greater than the maximum capacity of the oil transmission system (3.5 tons/min). The Disjunctive rule does not generate any conflicts and does not reject any of the information asserted by the sources. As such, no normalization procedure is required. The drawback of this method is that it yields a less precise result.

However, both Dempster's rule and the Disjunctive rule assume that the experts are independent. The Cautious rule is commutative, associative and idempotent. The property of idempotence makes it suitable for combining non-distinct items of evidence (i.e evidence from dependent experts). In Table XIV we can see that using the Cautious rule the oil transmission system may (but we cannot be sure) meet the system availability requirement $(A>0.6)$ if the demand is lower than $2(A=[0.499575,0.642124]$ for $w=1.5)$. On the other hand, the expected output performance $E d=[1.425048,1.923970]$ is always 
lower than 2 tons/min.

\section{CONCLUSION}

The current work proves the applicability of D-S theory and TBM in determining the reliability indices of MSSs in the presence of both aleatory and epistemic uncertainties. New definitions are given for MSS reliability indices in the framework of TBM. In particular, in the case of aleatory uncertainty, the same reliability indices are obtained for the UGF and TBM methods. Our results show that the proposed model might be used in practical situations when there is a need to take into account epistemic uncertainties and experts' opinions. The use of several combination rules in the TBM framework is also discussed.

\section{APPENDIX}

\section{A. Formula of marginalization}

Consider a BBA $m^{\Omega_{x} \Omega_{y}}$ defined on the Cartesian product $\Omega_{x} \Omega_{y}$. The marginal BBA $m^{\Omega_{x} \Omega_{y} \downarrow \Omega_{x}}$ on $\Omega_{x}$ is defined by:

$$
\begin{gathered}
m^{\Omega_{x} \Omega_{y} \downarrow \Omega_{x}}(A)=\sum_{B \subseteq \Omega_{x} \Omega_{y} / \operatorname{Proj}\left(B \downarrow \Omega_{x}\right)=A} m^{\Omega_{x} \Omega_{y}}(B) \\
\forall A \subseteq \Omega_{x}
\end{gathered}
$$

Where $\operatorname{Proj}\left(B \downarrow \Omega_{x}\right)=\left\{x \in \Omega_{x} / \exists y \in \Omega_{y},(x, y) \in B\right\}$.

\section{B. Formula of vacuous extension}

Consider a BBA $m^{\Omega_{x}}$ defined on $\Omega_{x}$. Its vacuous extension on $\Omega_{x} \Omega_{y}$ is defined by:

$$
m^{\Omega_{x} \uparrow \Omega_{x} \Omega_{y}}(B)= \begin{cases}m^{\Omega_{x}}(A) & \text { if } \quad B=A \times \Omega_{y} \\ 0 & \text { otherwise. }\end{cases}
$$

$$
\forall A \subseteq \Omega_{x}
$$




\section{Formulas of combination rules}

The Conjunctive (a) and Disjunctive (1) rules are defined by:

$$
\begin{aligned}
& m_{i @ j}^{\Omega}(H)=\sum_{A \cap B=H, \forall A, B \subseteq \Omega} m_{i}^{\Omega}(A) m_{j}^{\Omega}(B), \forall H \subseteq \Omega \\
& m_{i @ j}^{\Omega}(H)=\sum_{A \cup B=H, \forall A, B \subseteq \Omega} m_{i}^{\Omega}(A) m_{j}^{\Omega}(B), \forall H \subseteq \Omega
\end{aligned}
$$

Dempster's rule is given by:

$$
m_{i \oplus j}^{X}(H)=\frac{\sum_{A \cap B=H, \forall A, B \subseteq X} m_{i}^{X}(A) m_{j}^{X}(B)}{1-\sum_{A \cap B=\emptyset, \forall A, B \subseteq X} m_{i}^{X}(A) m_{j}^{X}(B)}
$$

\section{REFERENCES}

[1] Almond, R. G. Graphical belief modeling. Chapmand and Hall, 1995.

[2] Aven, T. On performance-measures for multistate monotone systems. Reliability Engineering \& System Safety 41 (1993), 259-266.

[3] Aven, T. Risk analysis: A tool for expressing and communicating uncertainty. In Proceedings of European Safety and Reliability Conference (ESREL), Edinburgh (2000).

[4] AVEn, T. On the need for restricting the probabilistic analysis in risk assessments to variability. Risk Analysis 30 (2010), 354-360.

[5] AvEn, T. Interpretations of alternative uncertainty representations in a reliability and risk analysis context. Reliability Engineering \& System Safety 96 (2011), 353-360.

[6] Aven, T., AND Zio, E. Some considerations on the treatment of uncertainties in risk assessment for practical decision making. Reliability Engineering \& System Safety 96 (2011), 64-74.

[7] Bae, H. R., GRANDhi, R. V., AND CANFIElD, R. A. An approximation approach for uncertainty quantification using evidence theory. Reliability Engineering \& System Safety 86 (2004), 215-225.

[8] Barlow, R., ANd Wu, A. Coherent systems with multi-state elements. Mathematics of Operations Research 3 (1978), $275-281$

[9] Birnbaum, Z. W., Esary, J. D., And Saunders, S. C. Multi-component systems and structures and their reliability. Technometrics 3 (1961), 55-77.

[10] Brunelle, R., And KapUR, K. Review and classification of reliability measures for multistate and continuum models. IIE Transactions 31 (1999), 1171-1180.

[11] Caselton, W. F., And Luo, W. Decision making with imprecise probabilities: Dempster-shafer theory and applications. Water Resources Research 28 (1992), 3071-3083.

[12] Cheng, C.-H., And Mon, D.-L. Fuzzy system reliability analysis by interval of confidence. Fuzzy Sets and Systems 56, 1 (1993), 29-35. 
[13] Dempster, A., And Kong, A. Uncertain evidence and artificial analysis. Journal of Statistical Planing and Inference 20 (1988), 355-368.

[14] Dempster, A. P. Upper and lower probabilities induced by a multivalued mapping. Annals of Mathematical Statistics 38 (1967), 325-339.

[15] Denceux, T. Modeling vague beliefs using fuzzy-valued belief structures. Fuzzy Sets and Systems 116 (2000), 167-199.

[16] Deneux, T. Conjunctive and disjunctive combination of belief functions induced by nondistinct bodies of evidence. Artificial Intelligence 172 (2008), 234-264.

[17] Ding, Y., AND Lisnianski, A. Fuzzy universal generating functions for multi-state system reliability assessment. Fuzzy Sets and Systems 159, 3 (2008), 307-324.

[18] Ding, Y., AND Zuo, M. J. A framework for reliability approximation of multi- state weighted k-out-of-n systems. IEEE Transactions on Reliability 59 (2010), 297-308.

[19] Ding, Y., Zuo, M. J., Lisnianski, A., And Tian, Z. G. Fuzzy multi-state system: General definition and performance assessment. IEEE Transactions on Reliability 57 (2008), 589 - 594.

[20] Dubois, D., And Prade, H. The principle of minimum specificity as a basis for evidential reasoning. Uncertainty in knowledge-based systems (1987), 75-84.

[21] El-Neveihi, E., Prochan, F., And Setharaman, J. Multi-state coherent systems. Journal of Applied Probability 15 (1978), 675-688.

[22] Ferson, S., And Ginzburg, L. Different methods are needed to propagate ignorance and variability. Reliability Engineering \& System Safety 54 (1996), 133-144.

[23] Finetti, B. D. Theory of Probability. New York: Wiley \& Sons., 1974.

[24] G. J. Klir, And Folger, T. A. Fuzzy Sets, Uncertainty and Information. Englewood Cliffs. Prentice-Hall., 1999.

[25] Hoyland, A., And Rausand, M. System Reliability Theory, Models and Statistical Methods. Wiley, 1994.

[26] IshizUKa, M., FU, K., AND YaO, J. Inference procedures and uncertainty for the problem reduction method. Inform Sci 28 (1982), 179-206.

[27] KLETZ, T. A. Identifying and assessing process industry hazards. 4th Edition, Institution of chemical engineers, 1999.

[28] Kohlas, J., And Monney, P. A. A Mathematical Theory of Hints: An Approach to the Dempster-Shafer Theory of Evidence. 1995.

[29] Konar, A. Computational Intelligence: Principles, Techniques And Applications. Springer-Verlag Berlin and Heidelberg $\mathrm{GmbH} \&$ Co. K, 2005.

[30] Laplace, P. S. A Philosophical Essay on Probabilities. New York: Dover Publications Inc., 1814, English edition 1951.

[31] Levitin, G., AND Lisnianski, A. Importance and sensitivity analysis of multi state systems using the Universal Generating Function method. Reliability Engineering \& System Safety 65, 3 (1999), 271-282.

[32] Levitin, G., AND XING, L. Reliability and performance of multi state systems with propagated failures having selective effect. Reliability Engineering \& System Safety 95, 6 (2010), 655-661.

[33] Limbourg, P., And SaVi, R. Fault tree analysis in an early design stage using the Dempster-Shafer theory of evidence. Risk, Reliability and Societal Safety-Aven and vinnem, Taylor \& Francis Group, 2007. 
[34] Lisnianski, A. Extended block diagram method for a multi-state system reliability assessment. Reliability Engineering \& System Safety 92, 12 (2007), 1601-1607.

[35] Lisnianski, A., And Levitin, G. Multi-State System Reliability: Assessment, Optimization and Applications. World Scientific Publishing Co Pte Ltd, 2003.

[36] LuCAs, C., AND ARAABI, B. Generalization of the dempstershafer theory: A fuzzy valued measure. IEEE Transactions on Fuzzy Systems 7 (1999), 255-270.

[37] M. Drouin, G. Parry, J. Lehner, G. Martinez-Guridi, J. LaChance, and T. Wheeler. Guidance on the Treatment of Uncertainties Associated with PRAs in Risk-informed Decision making. Report, NUREG1855-V.1, 2009.

[38] Montero, J., Tejada, J., And Yanez, J. General structure functions. Kybernetes 23 (1994), 10-19.

[39] NAtVig, B. Multistate Systems Reliability Theory with Applications. Wiley, 2010.

[40] Oberkampf, W. L., Helton, J. C., Joslyn, C. A., Wojtkiewicz, S. F., And Ferson, S. Challenge problems: uncertainty in system response given uncertain parameters. Reliability Engineering \& System Safety 85 (2004), 11-19.

[41] Ogawa, H., AND Fu, K. An inexact inference for damage assessment of existing structures. Int J Man Mach Stud 22 (1985), 295-306.

[42] Paulos, J. A. The Mathematics of Changing Your Mind. New York Times (US)., 2011.

[43] Рнам, H. Handbook of Reliability Engineering. Springer, 2003.

[44] Pourret, O., Collet, J., And Bon, J.-L. Evaluation of the unavailability of a multi state-component system using a binary model. Reliability Engineering \& System Safety 64, 1 (1999), 13-17.

[45] Ramirez-Marquez, J. E., And Coit, D. W. A Monte-Carlo simulation approach for approximating multi-state twoterminal reliability. Reliability Engineering \& System Safety 87, 2 (2005), 253-264.

[46] Rosqvist, T. On the use of expert judgement in the qualification of risk assessment. Thesis, Helsinki University of Technology, Finland, 2003.

[47] RUSPINI, E. H. The logical foundations of evidential reasoning. Technical Note 408, SRI International, Menlo Park, California, US (1986).

[48] S. Pashazadeh, AND Sharifi, M. Reliability assessment under uncertainty using dempster-shafer and vague set theories. In Computational Intelligence for Measurement Systems and Applications, 2008. CIMSA 2008. 2008 IEEE International Conference on (2008), vol. 1, pp. 131-136.

[49] Sallak, M., Schön, W., And Aguirre, F. The Transferable Belief Model for reliability analysis of systems with data uncertainties and failure dependencies. Proceedings of the Institution of Mechanical Engineers, Part O: Journal of Risk and Reliability 40 (2010), 266-278.

[50] Shafer, G. A Mathematical Theory of Evidence. Princeton University Press, 1976.

[51] Simon, C., AND Weber, P. Evidential networks for reliability analysis and performance evaluation of systems with imprecise knowledge. IEEE Transactions on Reliability 58 (2009), 69-87.

[52] SMETS, P. The degree of belief in a fuzzy event. Information Sciences 25 (1981), 119.

[53] SMETs, P. The combination of evidence in the Transferable Belief Model. IEEE Transactions on Pattern Analysis and Machine Intelligence 12 (1990), 447-458. 
[54] Smets, P., And Kennes, R. The Transferable Belief Model. Artificial Intelligence 66 (1994), 191-243.

[55] U. K. RAKOWSKY, AND Gocht, U. Reasoning in reliability centred maintenance based on a Dempster-Shafer approach. Proceedings of the Institution of Mechanical Engineers, Part O: Journal of Risk and Reliability 222 (2008), 605-612.

[56] Ushakov, I., Levitin, G., And Lisnianski, A. Multi-state system reliability: From theory to practice. In Proceedings of 3 rd international conference on mathematical methods in reliability, pp 635638, Trondheim, Norway (2002).

[57] Ushakov, I. A. A Universal Generating Function. Soviet Journal of Computing System Science 5 (1986), 118-129.

[58] Ushakov, I. A. Handbook of Reliability Engineering. Wiley-Interscience, 1994.

[59] VENn, J. The Logic of Chance. 1866.

[60] Walley, P. Statistical reasoning with imprecise probabilities. New York : Chapman and Hall, 1991.

[61] Wood, A. P. Multistate block diagrams and fault trees. IEEE Transactions on Reliability 34 (1985), $236-240$.

[62] Xue, J., And Yang, K. Dynamic reliability analysis of coherent multistate systems. IEEE Transactions on Reliability 44 (1995), 253-264.

[63] YAGER, R. R. Generalized probabilities of fuzzy events fromfuzzy belief structures. Information Sciences 28 (1982), 45-62.

[64] YAger, R. R. On the Dempster-Shafer framework and new combination rules. Information Sciences 41 (1987), 93-137.

[65] YANG, M., TC, T. C., AND WU, K. Generalized belief function, plausibility function, and dempsters combinational rule to fuzzy sets. International Journal of Intelligent Systems 18 (2003), 925-937.

[66] Yen, J. Generalizing the dempstershafer theory to fuzzy sets. IEEE Transactions on Systems, Man and Cybernetics 20 (1990), 559-570.

[67] ZADEH, L. Fuzzy sets and information granularity. Advances in fuzzy set theory and application (1979), 318.

[68] Zimmermann, H.-J. An application-oriented view of modeling uncertainty. European Journal of Operational Research 122 (2000), 190-198.

[69] Zio, E., Marella, M., And Podofillini, L. A Monte Carlo simulation approach to the availability assessment of multi-state systems with operational dependencies. Reliability Engineering \& System Safety 92, 7 (2007), 871-882.

Mohamed Sallak is an Associate Professor with the Computer Engineering Department in Compiegne University of Technology in France. He received a Ph.D. degree from Polytechnic Institute of Lorraine in 2007. His current research interests concern reliability studies under epistemic and aleatory uncertainties and particularly Dempster-Shafer and Imprecise probabilities theories. 
Walter Schön got a degree in 1985 from Ecole Normale Supérieure in Paris, and received a doctorate in solid state physics from Université Pierre et Marie Curie (Paris) in 1989. He is currently a Professor with the Department of Information Processing Engineering at the Université de Technologie de Compiègne, France. His current research interests concern reliability engineering and safety critical computer systems.

Felipe Aguirre received a M.S degree in 2007 from ENIM in Metz in France. He is currently a Ph.D. student in Compiegne University of Technology and his research interests concern the use of Transferable Belief Model for reliability analysis of systems with data uncertainties. 